\title{
Nonlocal InSAR Filtering for High Resolution DEM Generation from TanDEM-X Interferograms
}

\author{
Gerald Baier*, Cristian Rossi*, Marie Lachaise*, Xiao Xiang Zhu* ${ }^{\dagger}$, Richard Bamler* $\ddagger$ \\ *German Aerospace Center (DLR), Remote Sensing Technology Institute (IMF), Wessling, \\ Germany \\ $\dagger$ Technical University of Munich, Signal Processing in Earth Observation (SiPEO), Munich, \\ Germany \\ $\ddagger$ Technical University of Munich, Chair of Remote Sensing Technology, Munich, Germany
}

November 23, 2017

\begin{abstract}
We investigate the feasibility of generating highly accurate digital elevation models (DEM) from TanDEM-X interferograms by using nonlocal filters for phase denoising. Some of the shortcomings of existing nonlocal filters that render them not applicable to our goal are briefly described and a new filter is proposed that alleviates these problems. The most significant new properties are addressing the slope dependent denoising performance of existing nonlocal InSAR filters and several measures to bolster denoising near edge-like features. We evaluate the proposed filter using synthetic interferograms and by visual inspection of a DEM generated from a TanDEM-X interferogram.
\end{abstract}

Index terms - DEM, InSAR denoising, nonlocal filtering

\section{Introduction}

Although the TanDEM-X mission already supplies a highly accurate digital elevation model (DEM) according to the specification outlined in Table 1, there is still interest for even more accurate DEMs of higher resolution. To satisfy this demand German Aerospace Center (DLR) is developing processing algorithms for a new product, the so-called HDEMs, short for highresolution DEM [1].

HDEMs rely on multiple additional acquisitions with larger baselines, providing a two-fold benefit: more acquisitions lower the noise level by averaging and additionally the larger baselines result in smaller height errors from phase noise. Thus an averaging phase filter with a smaller spatial extent compared to the default processing toolchain [2], [3] suffices to fulfill the vertical accuracy requirements and more of the original spatial

\begin{tabular}{ccc}
\hline & pixel spacing & relative vertical accuracy \\
\hline TanDEM-X & $12 \mathrm{~m}$ & $2 \mathrm{~m}($ slope $\leq 20 \%)$ \\
DEM & & $4 \mathrm{~m}($ slope $\geq 20 \%)$ \\
\hline $\begin{array}{c}\text { TanDEM-X } \\
\text { HDEM }\end{array}$ & $6 \mathrm{~m}$ & $0.8 \mathrm{~m}$ \\
\hline
\end{tabular}

Table 1: TanDEM-X DEM and HDEM resolution and accuracy requirements

resolution can be preserved.

We propose an alternative approach to obtain DEMs of higher quality. The mandatory phase denoising is performed by a nonlocal filter, instead of the currently employed, operationally efficient Boxcar filter. Nonlocal filters have proven their worth in the optical domain for denoising images and exhibit unprecedented noise suppression and detail preservation.

The goal is again to generate a digital elevation model which is comparable in quality to the HDEM as specified in Table 1 without any additional acquisitions. Our previous research [4] suggests that this is indeed feasible and led to improvements concerning the staircasing effect [5] and computational efficiency.

We would like to draw a clear distinction between the official HDEM and the DEM generated by nonlocal filtering, henceforth referred to as NLDEM, The NLDEM can not, by its very nature, provide an update to existing DEMs nor is it as sensitive to small perturbations due to the HDEM's much smaller height of ambiguity. The advantages are that no new acquisitions are necessary at the cost of vastly increased, but still manageable, computational complexity.

In this manuscript we propose a new nonlocal filter that addresses some of the shortcomings of existing 
ones and evaluate it using synthetic benchmarks and a real data set.

\section{Nonlocal InSAR Filtering}

Nonlocal filtering was first introduced for denoising optical images in [6], and was later adapted to other noise statistics. The most widely known nonlocal filters for InSAR are NL-InSAR[7] and NL-SAR[8], which both compute the similarity measure based on the noise statistics of SAR speckle and the interferometric phase.

The underlying principle of all nonlocal filters is their exploitation of inherent, not necessarily connected redundancies in natural images. For each pixel similar pixels are sought in its vicinity, the so-called search window, which is typically of size $21 \times 21$ to $31 \times 31$ pixels. There is no penalty for pixels that are farther away, which starkly contrasts nonlocal filters to bilateral filters or Gaussian smoothing. Similar pixels are detected by not only comparing each other but also their surrounding area, called patches. By doing so, nonlocal filters give far more greater weight to textures and structures, which helps to preserve details. Pixels in the search window are assigned a weight depending on their similarity to the center pixels, and its estimate is computed via a weighted mean

$$
\hat{u}_{\mathbf{x}}=\sum_{\mathbf{y} \in \partial_{\mathbf{x}}} w_{\mathbf{x}, \mathbf{y}} u_{\mathbf{y}}
$$

where $\mathbf{x}$ and $\mathbf{y}$ denote pixel coordinates, $u$ the input image and $\hat{u}$ its filtered estimate, $\partial_{\mathbf{x}}$ the search window at $\mathbf{x}$ and $w$ the weights.

\section{Proposed Filter}

In our previous research [4] we observed that existing nonlocal filters indeed provide significant quality improvements over existing TanDEM-X DEMs. Yet both evaluated filters NL-InSAR [7] and NL-SAR[8] suffer from undesired properties: NL-InSAR produces terrace-like artifacts for hilly terrain and NL-SAR tends to oversmooth small details, were phase changes are not accompanied by intensity changes. The goal of our proposed nonlocal InSAR filter is to produce a DEM comparable in quality to the HDEM, but without the aforementioned properties. A flow graph of the filter is depicted in Fig. 1. The following paragraphs will explain the reasoning for various design decisions.

If a patch exhibits an unusual amount of noise not many similar patches are found in its vicinity. The result being that such a patch is not denoised properly. A common solution is a two-stage approach [9], where

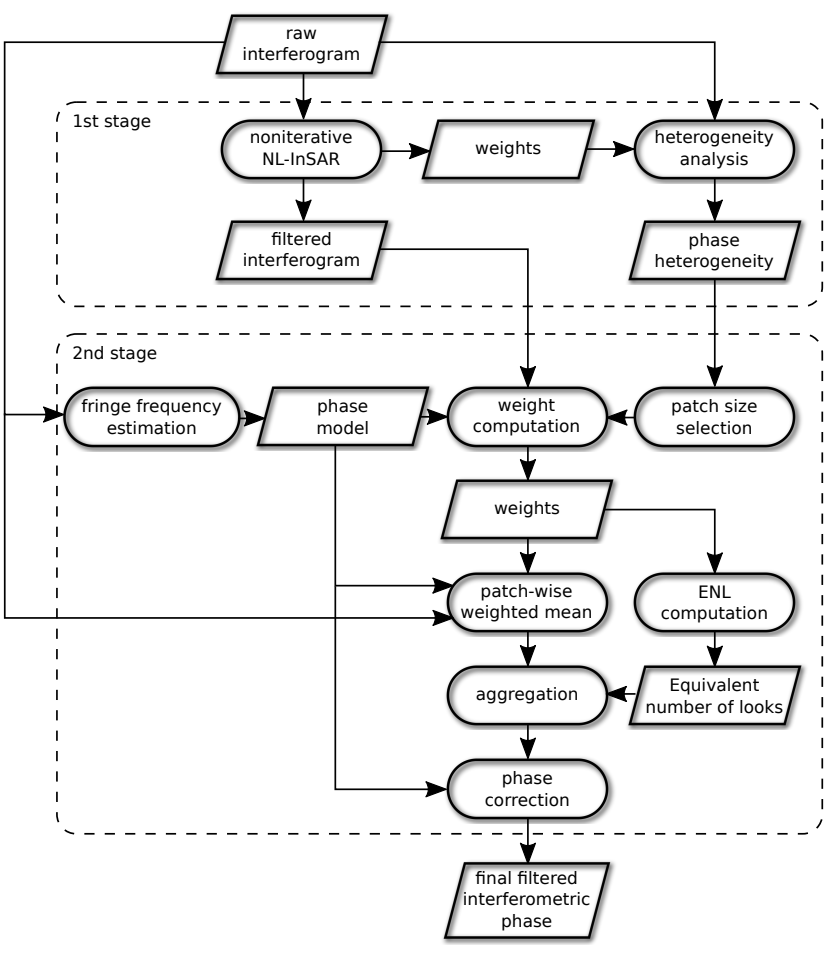

Figure 1: Flow graph of the proposed filter.

in the first step a so-called guidance image is generated by denoising the input image. In the second step the guidance image is used to compute the patch similarities, which are now more reliably estimated due to the reduced noise level. In our design the first stage of the proposed filter consists simply of the noniterative NL-InSAR filter.

For optimal denoising performance the parameters of a nonlocal filter - search window and patch dimensions, as well as the filtering strength - should be set adaptively [10]. We set the patch size depending on the phase heterogeneity index derived in [11]. Homogeneous areas are processed with a comparatively large patch size - up to $9 \times 9$, whereas for heterogeneous areas such as industrial or urban sites, the patch dimensions shrink down to $3 \times 3$. This way a more robust patch similarity can be computed in homogeneous areas, due to the larger patch size, while at the same time the rare patch effect is alleviated in heterogeneous areas.

In regions with high fringe frequencies, the deterministic topographic phase component causes the similarity of patches to drop quickly when moving away from the center patch. To counter this effect our proposed filter estimates the local fringe frequency inside the search window and later accounts for it when computing the similarities and the weighted means.

For the classic nonlocal filter a halo with high noise level manifests along edges due to the rare patch effect. To improve the denoising performance near edges our filter replaces the weighted mean Eq. (1) by a patch 
wise weighted mean

$$
\hat{\mathbf{u}}_{\mathbf{x}}=\sum_{\mathbf{y} \in \partial_{\mathbf{x}}} w_{\mathbf{x}, \mathbf{y}} \mathbf{u}_{\mathbf{y}}
$$

here $\mathbf{u}$ and $\hat{\mathbf{u}}$ represent patches centered at the respective positions and not just pixels. The final estimate is then obtained by aggregating overlapping patch estimates weighted by the equivalent number of looks of each, i.e. for denoising a pixel all patch estimates $\hat{\mathbf{u}}$ that contain the pixel are used and not only the one where the pixel is at the center. Especially near edges this procedure gives a more reliable estimate as a pixel near the edge also belongs to patches that do not include the edge, resulting in more similar pixels to be found.

\section{Experiments}

For our analysis we compare our proposed filter with NL-InSAR[7] and NL-SAR[8]. NL-SAR automatically picks the best parameters from a predefined set. For our analysis we chose the same set as in the original paper. NL-InSAR uses a search window of size $21 \times 21$, patches of size $7 \times 7$ and 5 iterations. The first stage of the proposed filter is identical to the noniterative version of NL-InSAR and also employs a search window of size $21 \times 21$ and a patch size of $7 \times 7$. The second stage selects the patch size $\{p \times p \mid p \in\{3,5,7,9\}\}$ depending on the heterogeneity and relies as well on a search window of size $21 \times 21$.

The two defining quality measures for denoising filters are detail preservation and noise reduction. Fig. 2 depicts the result of a Monte Carlo simulation of generated noisy interferograms for a step in phase and constant intensity and coherence. Both NL-InSAR and the proposed filter are able to preserve the sharp edge, whereas NL-SAR oversmoothes the result. NL-SAR integrates oversmoothing prevention, yet as it is intensity based it fails for this particular test scenario. We also see an increase of the standard deviation at the transition due to the rare patch effect, which is mitigated by the proposed solution.

To illustrate the staircasing effect synthetic terrain was generated using the diamond-square algorithm and a noisy interferogram was created. Fig. 3 shows in the top row the true phase and its noisy realization. The other rows show the phase estimates of the various nonlocal filters and their difference to the true phase. Clearly visible are plateau-like structures in the difference image for NL-InSAR, which would manifest as staircaes in the final DEM. Both NL-SAR and our proposed solution do not suffer from these artifacts. However NL-SAR produces a slightly more noisy estimate, especially in areas with denser fringes.

As a final example Fig. 4 shows shaded reliefs of digital elevation models with $3 \mathrm{~m}$ spacing generated from

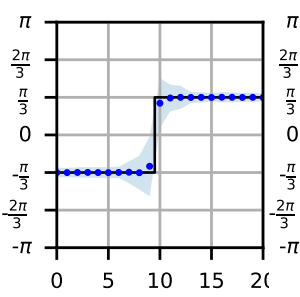

(a) NL-InSAR

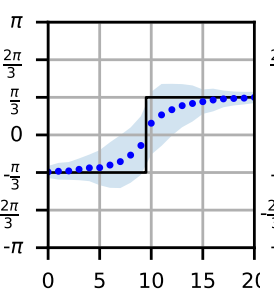

(b) NL-SAR

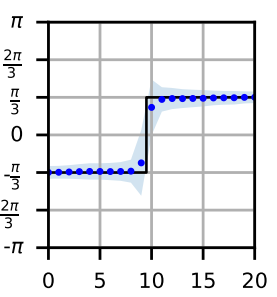

(c) proposed method
Figure 2: Estimated phase of a step function. The shaded blue area delineates \pm three times the estimate's standard deviation.

a single TanDEM-X interferogram using the different nonlocal filters. For comparison the result of a $5 \times 5$ boxcar filter and $6 \mathrm{~m}$ sampling, the configuration of the default TanDEM-X RawDEM, is also included. Evident is that more details are visible for the nonlocal filters and some of the terrace-like artifacts for NLInSAR. The shading does not do the NL-SAR result justice as it exaggerates small perturbations, which are not as severe as depicted. Yet we can clearly see that the fringe compensation of our proposed filter dramatically increases the performance in the mountainous region. Also obvious in the industrial area is that NLSAR and the proposed filter, which both employ a variable patch size, produce a less noisy DEM compared to NL-InSAR's result, which significantly suffers from the rare patch effect around buildings.

\section{Conclusion and Outlook}

We presented a nonlocal InSAR filter that addresses several open issues such as the staircasing effect or the slope dependent denoising performance. The intended goal of this filter is to produce a digital elevation model close to the HRTI-4 standard from TanDEM-X interferograms. We intent to perform a more thorough verification using LiDAR digital elevation models as well as DLR's HDEM.

\section{References}

[1] B. Wessel, M. Breunig, M. Bachmann, M. Huber, M. Martone, M. Lachaise, T. Fritz, and M. Zink, "Concept and first example of TanDEM-X highresolution DEM," in EUSAR 2016; Proc. of, Jun. 2016.

[2] C. Rossi, F. R. Gonzalez, T. Fritz, N. YagueMartinez, and M. Eineder, "TanDEM-X calibrated raw DEM generation," ISPRS Journal of Photogrammetry and Remote Sensing, vol. 73, pp. 12-20, 2012. 

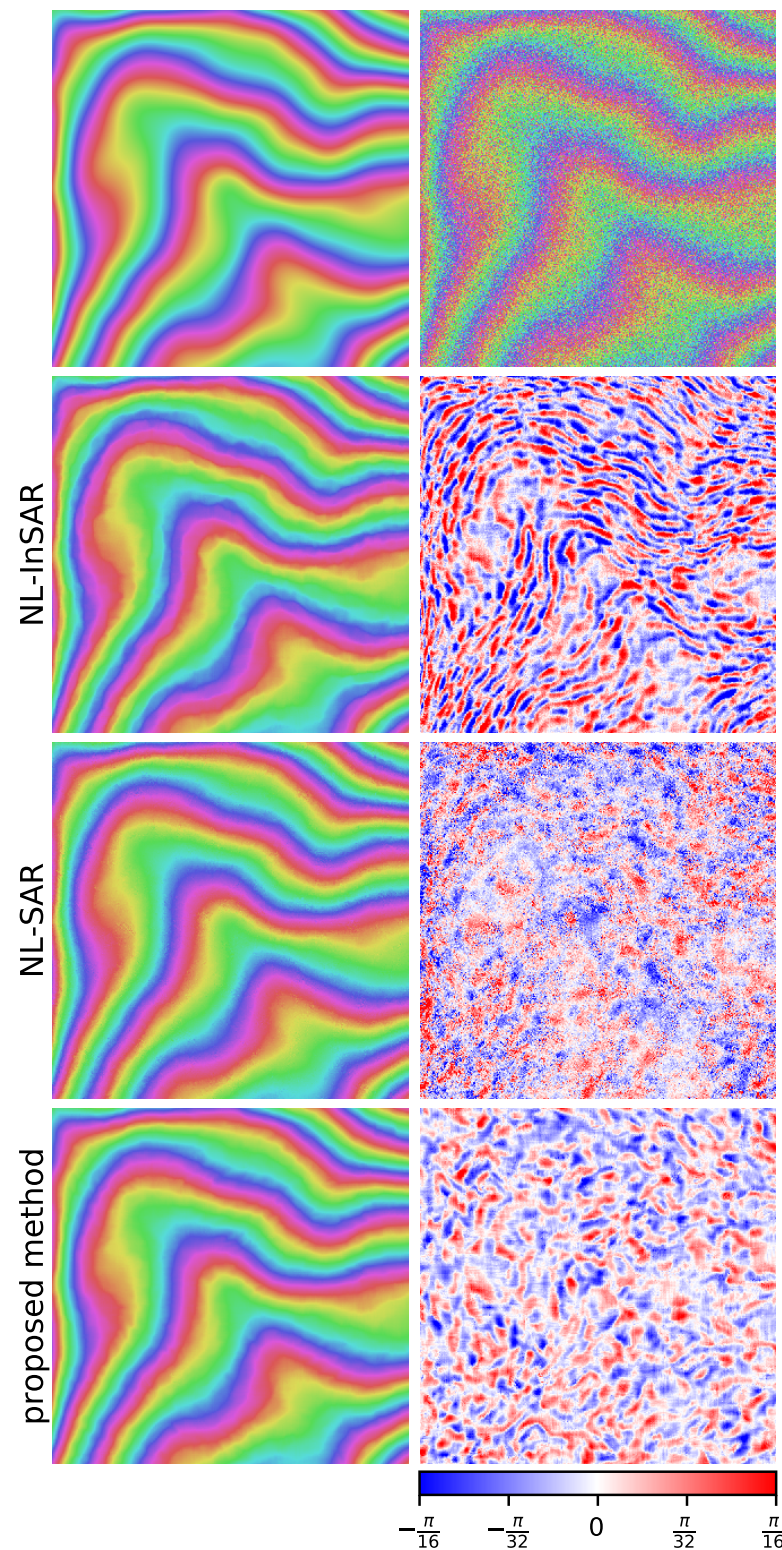

Figure 3: Phase estimates and differences for a synthetically generated interferogram. The phase estimate of NL-InSAR shows a distinct staircase-like pattern.

[3] T. Fritz, C. Rossi, N. Yague-Martinez, F. Rodriguez-Gonzalez, M. Lachaise, and H. Breit, "Interferometric processing of TanDEM-X data," in Geoscience and Remote Sensing Symposium (IGARSS), 2011 IEEE International, Jul. 2011, pp. 2428-2431.

[4] X. X. Zhu, R. Bamler, M. Lachaise, F. Adam, Y. Shi, and M. Eineder, "Improving TanDEM-X DEMs by Non-local InSAR Filtering," in EUSAR 2014; Proc. of, Jun. 2014.

[5] G. Baier, X. X. Zhu, M. Lachaise, H. Breit, and R. Bamler, "Nonlocal InSAR filtering for DEM generation and addressing the staircasing effect," in EUSAR 2016; Proc. of, Jun. 2016.

[6] A. Buades, B. Coll, and J.-M. Morel, "A nonlocal algorithm for image denoising," in IEEE Computer Society Conference on Computer Vision and Pattern Recognition, 2005. CVPR 2005, vol. 2, Jun. 2005, pp. 60-65.

[7] C.-A. Deledalle, L. Denis, and F. Tupin, "NL-InSAR: Nonlocal interferogram estimation," IEEE Transactions on Geoscience and Remote Sensing, vol. 49, no. 4, pp. 1441-1452, Apr. 2011.

[8] C.-A. Deledalle, L. Denis, F. Tupin, A. Reigber, and M. Jäger, "NL-SAR: A Unified Nonlocal Framework for Resolution-Preserving (Pol)(In)SAR Denoising," IEEE Transactions on Geoscience and Remote Sensing, vol. 53, no. 4, pp. 2021-2038, Apr. 2015.

[9] J. Salmon, R. Willett, and E. Arias-Castro, "A two-stage denoising filter: The preprocessed yaroslavsky filter," in 2012 IEEE Statistical Signal Processing Workshop (SSP), Aug. 2012, pp. 464-467.

[10] V. Duval, J.-F. Aujol, and Y. Gousseau, "A bias-variance approach for the non-local means," SIAM Journal on Imaging Sciences, vol. 4, no. 2, pp. 760-788, Jan. 2011.

[11] J. S. Lee, K. P. Papathanassiou, T. L. Ainsworth, M. R. Grunes, and A. Reigber, "A new technique for noise filtering of SAR interferometric phase images," IEEE Transactions on Geoscience and Remote Sensing, vol. 36, no. 5, pp. 1456-1465, Sep. 1998. 


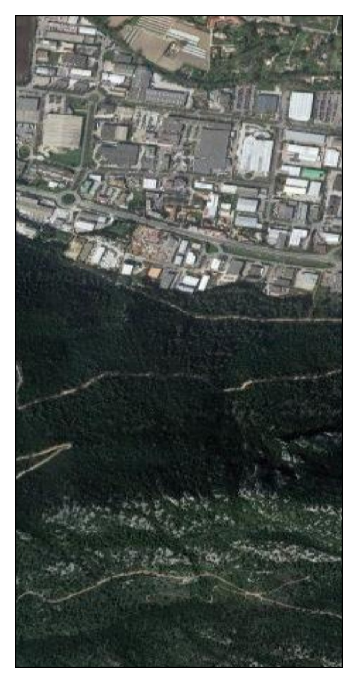

(a) optical image (c) Google

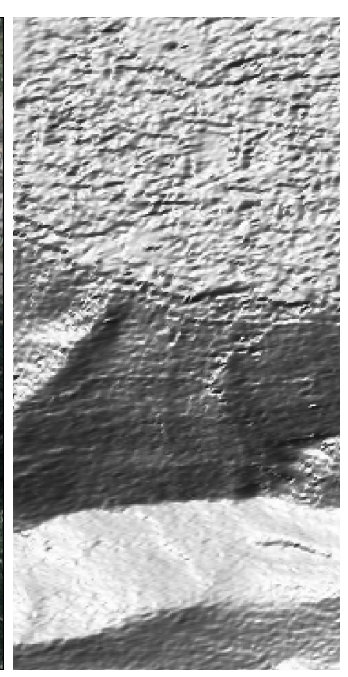

(b) boxcar

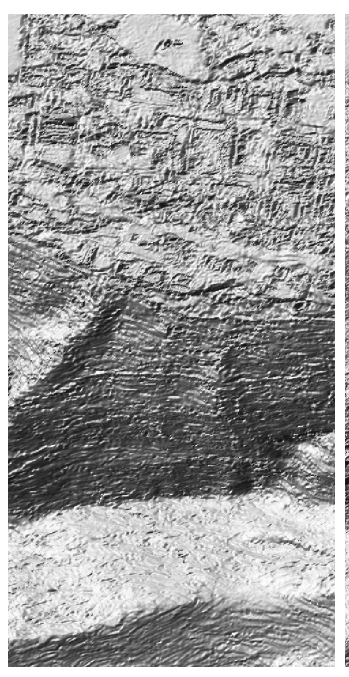

(c) NL-InSAR

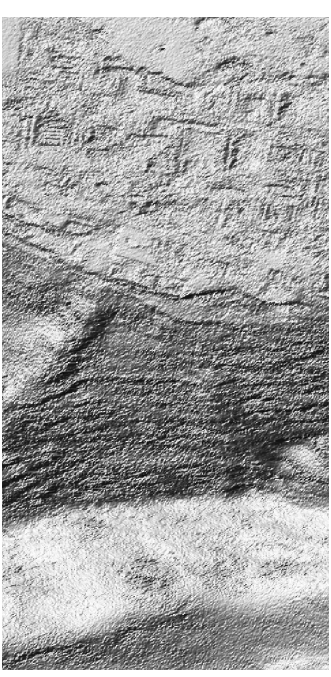

(d) NL-SAR

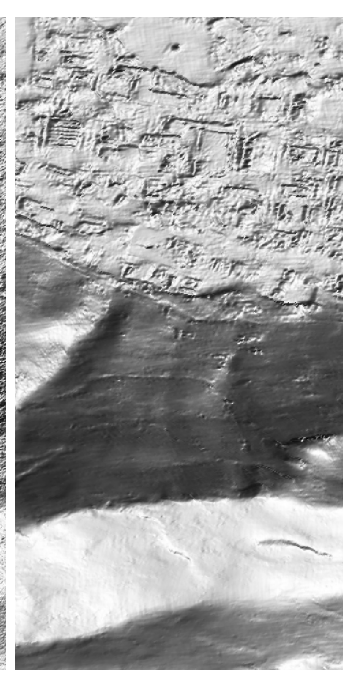

(e) proposed method

Figure 4: Digital elevation models generated from a single TanDEM-X interferogram using a $5 \times 5$ boxcar filter, the default ITP denoising filter and $6 \mathrm{~m}$ sampling and the previously described nonlocal filters with $3 \mathrm{~m}$ sampling. 